\title{
Protective effects of Radix Pseudostellariae polysaccharides against exercise-induced oxidative stress in male rats
}

\author{
ZICHAO CHEN ${ }^{1}$, SHANSHAN LI $^{1}$, XIAOQIN WANG ${ }^{2}$ and CHUAN LONG ZHANG ${ }^{3}$ \\ ${ }^{1}$ Department of Physical Education, Sichuan University, Chengdu, Sichuan 610064; ${ }^{2}$ West China Second University Hospital, \\ Sichuan University, Chengdu, Sichuan 610041; ${ }^{3}$ Luoyang Institute of Science and Technology, \\ Luoyang, Henan 471023, P.R. China
}

Received December 8, 2012; Accepted January 21, 2013

DOI: $10.3892 /$ etm.2013.942

\begin{abstract}
The main purpose of this study was to examine the effect of Radix Pseudostellariae polysaccharides (RPPs) against swimming exercise-induced oxidative stress in male rats. A total of 40 male Wistar rats were randomized into four groups: the control (C), low-dose RPP supplementation (LRS), medium-dose RPP supplementation (MRS) and high-dose RPP supplementation (HRS) groups. The control group received saline solution and the supplementation groups received different doses of RPPs $(100,200$ and $400 \mathrm{mg} / \mathrm{kg}$ body weight, respectively). The animals were medicated orally and daily for 28 days. On day 28 , the rats were made to swim until exhausted. The exhaustive swimming time and various biochemical parameters, including blood lactate, hemoglobin, catalase (CAT), superoxide dismutase (SOD), glutathione peroxidase (GSH-Px) and malondialdehyde (MDA), were measured. The results showed that RPP supplementation elevates the exercise tolerance and decreases the blood lactate level of rats following exhaustive swimming exercise. RPP supplementation augments the levels of hemoglobin and antioxidant enzymes (CAT, SOD and GSH-Px), and effectively decreases the MDA content of the skeletal muscle of rats, which suggests that RPP supplementation has a protective effect against exercise-induced oxidative stress.
\end{abstract}

\section{Introduction}

During the past few decades, a series of studies have addressed the association of strenuous physical exercise with increased oxygen uptake and generation of reactive oxygen species (ROS) in various mammals (1-4). In general, the body has adequate antioxidant reserves to cope with the production of ROS under

Correspondence to: Professor Shanshan Li, Department of Physical Education, Sichuan University, Wangjiang Road 29, Chengdu, Sichuan 610064, P.R. China

E-mail: sli37@yahoo.cn; czc5233@163.com

Key words: Radix Pseudostellariae polysaccharides, swimming exercise, oxidative stress, rats physiological conditions. The antioxidant system consists of antioxidant vitamins, glutathione and thiols, and antioxidant enzymes. Each of these antioxidants plays a unique role within the cell and complements the others functionally (5). However, when ROS levels exceed the normal physiological coping range during strenuous physical exercise, the accumulation of ROS and a reduction in antioxidant status may result. This scenario increases oxidative stress and leads to modifications of lipid and protein structures that consequently compromise the cellular functions in tissue $(6,7)$. To increase the body's antioxidant potential and to decrease levels of oxidative stress, it has been recommended that individuals increase their intake of dietary antioxidants. Dietary antioxidants interact with endogenous antioxidants to form a cooperative antioxidant network (8).

Radix Pseudostellariae, the root of Pseudostellaria heterophylla (Miq.) Pax. known as 'Tongshen' or 'Taizishen', has a long history of medicinal use in China. As a traditional Chinese medicine, it is frequently used to treat disease, in particular as a lung and spleen tonic $(9,10)$. A number of studies concerning Radix Pseudostellariae, including its chemical components and relevant pharmacological properties, have been performed. Radix Pseudostellariae polysaccharides (RPPs) have displayed clear anti-infectious, anti-inflammatory, hypoglycemic, hypolipidemic and immunomodulating activities (11-14). Studies have also shown that RPPs exhibit strong antioxidant activities (15), which suggests that they are beneficial in counteracting exercise-induced oxidative stress. However, the effects of RPPs on exercise-induced oxidative stress have not been investigated thus far. Therefore, in the present study, we investigated the effects of RPP supplementation against the exercise-induced oxidative stress of forced swimming in male rats.

\section{Materials and methods}

Materials and chemicals. The dried Radix Pseudostellariae (native to Shandong herbal medicines planting base, China) was purchased from Tongjitang Herb Shop (Chengdu, China). The authenticity of the plant was confirmed by Dr MF Li, a botanist at Sichuan University (Chengdu, China), and a voucher specimen was deposited in the Herbarium of Sichuan University. The dried Radix Pseudostellariae was ground with an electric mixer prior to extraction. The assay kits for blood 
lactate, hemoglobin, catalase (CAT), superoxide dismutase (SOD), glutathione peroxidase (GSH-Px) and malondialdehyde (MDA) were purchased from Jianchen Bioengineering Institute (Nanjing, China). Other chemicals and biochemicals were of analytical grade and were purchased from Sigma Chemical Co. (St. Louis, MO, USA) and Changsheng Pharmaceutical Co. (Chengdu, China) unless otherwise indicated.

Experiment animals. Healthy male Wistar rats with an average mass of 225-250 g were obtained from Sichuan Research Animal Center (Chengdu, China). A standard pellet diet and water were provided ad libitum. The animals were housed in a temperature-controlled room at $21-23^{\circ} \mathrm{C}$ and maintained on a $12 \mathrm{~h}$ light : $12 \mathrm{~h}$ dark cycle. All animals received humane care according to the guidelines of the Guidebook for the Care and Use of Laboratory Animals (16). The study protocol was approved by the Animal Research Ethics Committee at Sichuan University (Chengdu, China).

Preparation of RPPs. The preparation of RPPs was carried out according to the literature $(17,18)$. In brief, the dried Radix Pseudostellariae was ground into powder. The powder (400 g) was extracted three times by refluxing with $80 \%$ ethanol (1 liter) at $90^{\circ} \mathrm{C}$ for $2-3 \mathrm{~h}$ each time. After filtration, the dregs were extracted again three times with water $(1.5$ liter $)$ at $90^{\circ} \mathrm{C}$ for 2-3 h each time. The extracted solution was condensed to $400 \mathrm{ml}$ and deproteinated using the Sevag method. The solution was then added to absolute ethanol until the ethanol concentration was $80 \%$ and kept overnight, followed by filtration. The precipitate was dissolved with water $(100 \mathrm{ml})$ and then absolute ethanol was added until the ethanol concentration was $80 \%$, the solution was filtrated and this method was repeated once again. The precipitate was washed with $95 \%$ ethanol, absolute ethanol and acetone by turns, and then dried at $50^{\circ} \mathrm{C}$.

Experimental protocol. A total of 40 healthy male Wistar rats were randomized into four equal groups based on body weight following one week where rats acclimated to the new environment: the control (C), low-dose RPP supplementation (LRS), medium-dose RPP supplementation (MRS) and high-dose RPP supplementation (HRS) groups. The $\mathrm{C}$ group received saline solution and the supplementation groups received different doses of RPPs (100, 200 and $400 \mathrm{mg} / \mathrm{kg}$ body weight, respectively). The treatments were administered orally and daily for 28 days.

Following the final supplementation with RPPs or saline solution, the rats were allowed to rest for $30 \mathrm{~min}$. The rats were then removed for the exhaustive swimming exercise. The details of this apparatus have been reported previously (19). A acrylic plastic pool $(90 \times 60 \times 60 \mathrm{~cm})$ was filled with water to a depth of $40 \mathrm{~cm}$ and maintained at $28 \pm 1^{\circ} \mathrm{C}$. The rats were forced to swim in the water, and the endurance was defined as the time active swimming was maintained until the animal submerged in the water without movement. To diminish stress, all rats had been accustomed to swimming with repeated short-term swimming sessions for a week prior to experimentation.

Analysis of biochemical parameters. At the end of the swimming test, the rats were anesthetized with pentobarbital sodium

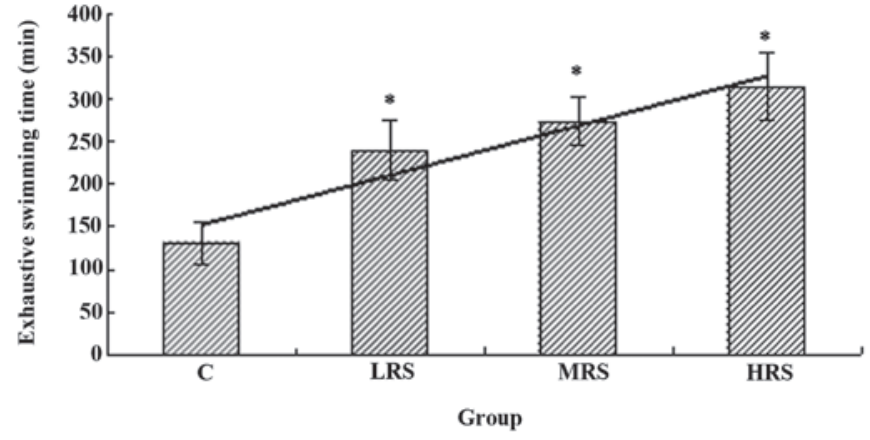

Figure 1. Effects of RPP supplementation on the exhaustive swimming times of rats. Data are presented as the mean $\pm \mathrm{SD}$ of ten rats per group. ${ }^{*} \mathrm{P}<0.05$, compared with the control (C) group. RPP, Radix Pseudostellariae polysaccharide; LRS, low-dose RPP supplementation; MRS, medium-dose RPP supplementation; HRS, high-dose RPP supplementation.

(5 mg/100 g body weight, i.p.). Blood was obtained from the orbital sinus for lactate and hemoglobin level measurements. Hindlimb skeletal muscle was rapidly removed and homogenized immediately in ice-cold $10 \% \mathrm{KCl}$ solution $(10 \mathrm{ml} / \mathrm{g}$ of tissue) using a teflon/glass homogenizer. The suspension was centrifuged at $671 \mathrm{xg}$ at $4^{\circ} \mathrm{C}$ for $10 \mathrm{~min}$ and the clear supernatant was used for CAT, SOD, GSH-Px and MDA level measurements. All biochemical parameters were determined using commercial kits following the manufacturer's recommended instructions.

Statistical analysis. The data are expressed as the mean \pm SD. Statistical comparisons were compared by one-way analysis of variance (ANOVA). $\mathrm{P}<0.05$ was considered to indicate a statistically significant result.

\section{Results and Discussion}

Effects of RPP supplementation on the exhaustive swimming times of rats. Exhaustive swimming was selected as a model of physical exercise since muscle trauma caused by other types of physical exercise, including prolonged running on a treadmill, exercise stimulated by electric shock and plyometric contractions, may be avoided (20,21). As shown in Fig. 1, exhaustive swimming times in all the RPP supplementation groups were significantly longer compared with that of the $\mathrm{C}$ group $(\mathrm{P}<0.05)$. These results indicate that RPP supplementation is able to elevate exercise endurance.

Effects of RPP supplementation on the blood lactate levels of rats. Lactate serves as an energy source in highly oxidative tissues. Numerous organs, including the liver and heart, and tissues such as skeletal muscle, aid the removal of lactate from the blood, but intense exercise increases lactate production (22). As shown in Fig. 2, the blood lactate level in each of the RPP supplementation groups was significantly lower compared with that of the $\mathrm{C}$ group $(\mathrm{P}<0.05)$. These results indicated that RPP supplementation effectively attenuates the increase of blood lactate, which may be responsible for the improvement in exercise endurance.

Effects of RPP supplementation on the hemoglobin levels of rats. Hemoglobin is the main component of erythrocytes. The 


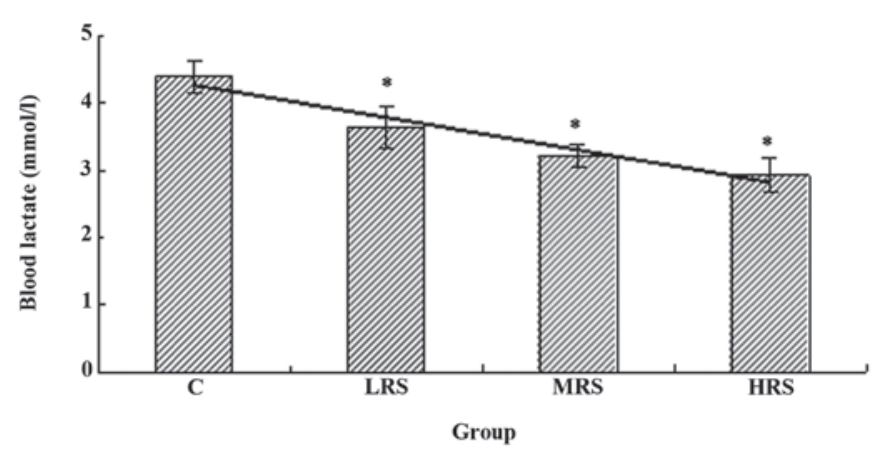

Figure 2. Effects of RPP supplementation on the blood lactate level of rats. Data are presented as the mean $\pm \mathrm{SD}$ of ten rats per group. ${ }^{*} \mathrm{P}<0.05$, compared with the control (C) group. RPP, Radix Pseudostellariae polysaccharide LRS, low-dose RPP supplementation; MRS, medium-dose RPP supplementation; HRS, high-dose RPP supplementation.

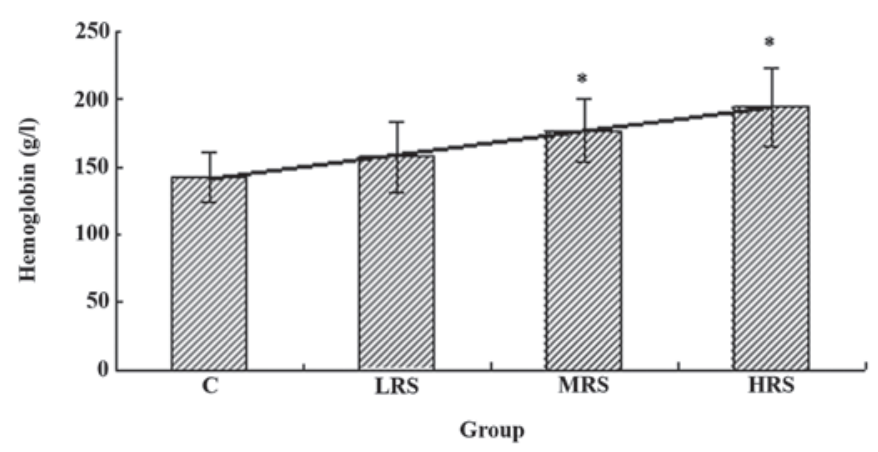

Figure 3. Effects of RPP supplementation on the hemoglobin level of rats Data are presented as the mean \pm SD of ten rats per group. ${ }^{*} \mathrm{P}<0.05$, compared with the control (C) group. RPP, Radix Pseudostellariae polysaccharide; LRS, low-dose RPP supplementation; MRS, medium-dose RPP supplementation; HRS, high-dose RPP supplementation.

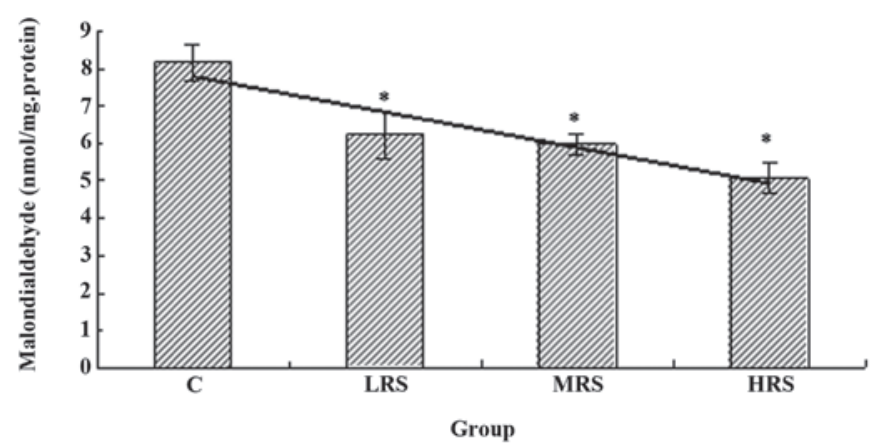

Figure 4. Effects of RPP supplementation on the MDA content of skeletal muscle in rats. Data are presented as the mean $\pm \mathrm{SD}$ of ten rats per group. ${ }^{*} \mathrm{P}<0.05$, compared with the control (C) group. RPP, Radix Pseudostellariae polysaccharide; LRS, low-dose RPP supplementation; MRS, medium-dose RPP supplementation; HRS, high-dose RPP supplementation.

improvement of cardiopulmonary function and increase of oxygen supply to tissues caused by an increase in hemoglobin levels are commonly stated to be major factors that increase endurance capacity (23). As shown in Fig. 3, the hemoglobin levels in the MRS and HRS groups were significantly higher compared with that of the $\mathrm{C}$ group $(\mathrm{P}<0.05)$. Although the hemoglobin level in the LRS group was also increased, no significant difference was observed $(\mathrm{P}>0.05)$. These results
Table I. Effects of RPP supplementation on the antioxidant enzyme content of skeletal muscle in rats.

\begin{tabular}{lccc}
\hline Group & $\begin{array}{c}\text { CAT } \\
(\mathrm{U} / \mathrm{mg} \text { protein })\end{array}$ & $\begin{array}{c}\text { SOD } \\
(\mathrm{U} / \mathrm{mg} \text { protein })\end{array}$ & $\begin{array}{c}\text { GSH-Px } \\
(\mathrm{U} / \mathrm{mg} \text { protein })\end{array}$ \\
\hline C & $3.16 \pm 0.32$ & $101.31 \pm 11.42$ & $6.57 \pm 1.38$ \\
LRS & $3.33 \pm 0.41$ & $148.53 \pm 10.78^{\mathrm{a}}$ & $9.86 \pm 1.85^{\mathrm{a}}$ \\
MRS & $4.15 \pm 0.39^{\mathrm{a}}$ & $162.87 \pm 13.46^{\mathrm{a}}$ & $12.39 \pm 1.16^{\mathrm{a}}$ \\
HRS & $4.48 \pm 0.46^{\mathrm{a}}$ & $169.52 \pm 12.24^{\mathrm{a}}$ & $16.34 \pm 1.72^{\mathrm{a}}$ \\
\hline
\end{tabular}

Data are the mean \pm SD of ten rats per group. ${ }^{\mathrm{a}} \mathrm{P}<0.05$, compared with the control (C) group. RPP, Radix Pseudostellariae polysaccharides; CAT, catalase; SOD, superoxide dismutase; GSH-Px, glutathione peroxidase; LRS, low-dose RRP supplementation; MRS, medium-dose RRP supplementation; HRS, high-dose RRP supplementation.

indicated that RPP supplementation may influence the supply of oxygen to tissues by hemoglobin, and may contribute to the improvement in exercise endurance.

Effects of RPP supplementation on the MDA content of rat skeletal muscle. Lipid peroxidation represents oxidative tissue damage caused by hydrogen peroxide, superoxide anions and hydroxyl radicals, resulting in structural alteration of the membrane, release of cell and organelle content and loss of essential fatty acids with formation of cytosolic aldehyde and peroxide products (24). MDA is a secondary product generated during the oxidation of polyunsaturated fatty acids, which has been frequently measured as an indicator of lipid peroxidation and oxidative stress in vivo (25). Numerous studies have observed that strenuous physical exercise induces increases in the MDA concentration in tissues $(24,26,27)$. As shown in Fig. 4, the MDA content in all three RPP supplementation groups was significantly lower compared with that of the $\mathrm{C}$ group $(\mathrm{P}<0.05)$. These results indicate that RPP supplementation effectively reduces lipid peroxidation.

Effects of RPP supplementation on the antioxidant enzyme content of rat skeletal muscle. CAT, SOD and GSH-Px are regarded as the first line of defense by the antioxidant enzyme system against ROS generated during exhaustive exercise (28). SOD catalyzes the dismutation of superoxide into oxygen and hydrogen peroxide. GSH-Px is a selenoenzyme which catalyzes the reduction of hydroperoxides at the expense of reduced glutathione. CAT is a primary antioxidant defense component that catalyzes the decomposition of hydrogen peroxide to water, sharing this function with GSH-Px (29). As shown in Table I, the CAT contents in the MRS and HRS groups were significantly higher compared with that of the $\mathrm{C}$ group $(\mathrm{P}<0.05)$. Although the CAT content in the LRS group was also increased, no significant difference was observed (P>0.05). The SOD and GSH-Px contents in the RPP supplementation groups were significantly higher compared with that of the $\mathrm{C}$ group $(\mathrm{P}<0.05)$. These results indicate that RPP supplementation upregulated antioxidant enzymes to protect against oxidative stress-induced injury during exhaustive exercise. 
From the present findings, we conclude that RPP supplementation elevates the exercise tolerance and decreases the blood lactate level of rats following exhaustive swimming exercise. RPP supplementation augments the levels of hemoglobin and antioxidant enzymes and effectively decreases the MDA content in the skeletal muscle, which suggests that RPP supplementation possesses protective effects against swimming-induced oxidative stress. Our data relates to rats, therefore future studies using different subjects, possibly of different sporting backgrounds, are required to extend these findings.

\section{Acknowledgements}

This study was supported by by a Sichuan University CrossDisciplinary Research Project grant (No. SKQY201109)

\section{References}

1. Alessio HM: Exercise-induced oxidative stress. Med Sci Sports Exerc 25: 218-224, 1993.

2. Bejma J and Ji LL: Aging and acute exercise enhance free radical generation in rat skeletal muscle. J Appl Physiol 87: 465-470, 1999.

3. Akhtar M, Pillai KK and Vohora D: Effect of thioperamide on modified forced swimming test-induced oxidative stress in mice. Basic Clin Pharmacol Toxicol 97: 218-221, 2005.

4. Pepe H, Balci SS, Revan S, Akalin PP and Kurtoğlu F: Comparison of oxidative stress and antioxidant capacity before and after running exercises in both sexes. Gend Med 6: 587-595, 2009.

5. Banerjee AK, Mandal A, Chanda D and Chakraborti S: Oxidant, antioxidant and physical exercise. Mol Cell Biochem 253: 307-312, 2003.

6. Viña J, Gomez-Cabrera MC, Lloret A, Marquez R, Miñana JB, Pallardó FV and Sastre J: Free radicals in exhaustive physical exercise: mechanism of production, and protection by antioxidants. IUBMB Life 50: 271-277, 2000.

7. Taysi S, Oztasan N, Efe H, Polat MF, Gumustekin K, Siktar E, Canakci E, Akcay F, Dane S and Gul M: Endurance training attenuates the oxidative stress due to acute exhaustive exercise in rat liver. Acta Physiol Hung 95: 337-347, 2008.

8. Powers SK, DeRuisseau KC, Quindry J and Hamilton KL: Dietary antioxidants and exercise. J Sports Sci 22: 81-94, 2004.

9. Gong Z, Dai Y, Ma H, Wang Z and Yu G: The effect of Radix Pseudostellariae from 8 habitats on spleen-deficiency and immunologic function. Zhong Yao Cai 24: 281-282, 2001 (In Chinese).

10. Lin H, Chen Q, Zhao J and Zhou P: Determination of free amino acid content in Radix Pseudostellariae using near infrared (NIR) spectroscopy and different multivariate calibrations. J Pharm Biomed Anal 50: 803-808, 2009.

11. Wong CK, Leung KN, Fung KP and Choy YM: The immunostimulating activities of anti-tumor polysaccharides from Pseudostellaria heterophylla. Immunopharmacology 28: 47-54, 1994.

12. Ng TB, Liu F and Wang HX: The antioxidant effects of aqueous and organic extracts of Panax quinquefolium, Panax notoginseng, Codonopsis pilosula, Pseudostellaria heterophylla and Glehnia littoralis. J Ethnopharmacol 93: 285-288, 2004.
13. Cai J, Li X, Chen, X et al: The immune effects of crude extract of Pseudostellaria polysaccharide in mice. Journal of Fujian College of Traditional Chinese Medicine 15: 33-35, 2005 (In Chinese).

14. Li J and Fu Y: Effect of IgY, Coptis chinensis and Radix Pseudostellariae on gastric mucous membrane in mice with Helicobacter pylori infection. Chinese Journal of Clinical Rehabilitation 10: 78-80, 2006 (In Chinese).

15. Wang WK, Jia JR. Din W and Liu HN: Recent advances in studies on Pseudostellariae Radix. Chinese Journal of Experimental Traditional Medical Formulae 17: 264-267, 2011 (In Chinese).

16. (NRC) Institute of Laboratory Animal Resources, Commission on Life Sciences, National Research Council: Guide for the Care and Use of Laboratory Animals. National Academies Press, Washington, DC, pp 11-45, 1996.

17. Chen YY, Ding Y, Wang W, Wang RF, Su H and Du LJ: Determination of polysaccharide in Radix pseudostellariae extract by size-exclusion high-performance liquid chromatography. Tsinghua Sci Tech 12: 389-393, 2007.

18. Sheng R, Xu X, Tang Q, Bian D, Li Y, Qian C, He X, Gao X, Pan R, Wang C, Luo Y, Xia Y and Dai Y: Polysaccharide of Radix Pseudostellariae improves chronic fatigue syndrome induced by poly I:C in mice. Evid Based Complement Alternat Med 2011: 840516, 2011

19. Lee SP, Mar GY and Ng LT: Effects of tocotrienol-rich fraction on exercise endurance capacity and oxidative stress in forced swimming rats. Eur J Appl Physiol 107: 587-595, 2009.

20. Misra DS, Maiti R and Ghosh D: Protection of swimming-induced oxidative stress in some vital organs by the treatment of composite extract of Withania somnifera, Ocimum sanctum and Zingiber officinalis in male rat. Afr J Tradit Complement Altern Med 6: 534-543, 2009.

21. Ou PF and Zhang L: Stimulatory effects of soybean isoflavones on exercise performance. Int J Phys Sci 5: 2272-2277, 2010.

22. Wei W, Zheng LY, Yu MY, Jiang N, Yang ZR and Luo X: Antifatigue activity of extract form the submerged fermentation of Ganoderma Lucidum using Radix astragali as substrate. J Anim Plant Sci 6: 677-684, 2010.

23. Ikeuchi M, Yamaguchi K, Koyama T, Sono Y and Yazawa K: Effects of fenugreek seeds (Trigonella foenum greaecum) extract on endurance capacity in mice. J Nutr Sci Vitaminol (Tokyo) 52: 287-292, 2006.

24. Kato J, Ruram AA, Singh SS, Devi SB, Devi TI and Singh WG: Lipid peroxidation and antioxidant vitamins in urolithiasis. Indian J Clin Biochem 22: 128-130, 2007.

25. Li F, Tang H, Xiao F, Gong J, Peng Y and Meng X: Protective effect of salidroside from Rhodiolae Radix on diabetes-induced oxidative stress in mice. Molecules 16: 9912-9924, 2011.

26. Kanter MM, Nolte LA and Holloszy JO: Effects of an antioxidant vitamin mixture on lipid peroxidation at rest and postexercise. J Appl Physiol 74: 965-969, 1993.

27. Zhang M, Izumi I, Kagamimori S, Sokejima S, Yamagami T, Liu Z and Qi B: Role of taurine supplementation to prevent exercise-induced oxidative stress in healthy young men. Amino Acids 26: 203-207, 2004.

28. Huang CC, Lin TJ, Lu YF, Chen CC, Huang CY and Lin WT: Protective effects of L-arginine supplementation against exhaustive exercise-induced oxidative stress in young rat tissues. Chin J Physiol 52: 306-315, 2009.

29. Cotgreave IA, Moldéus P and Orrenius S: Host biochemical defense mechanisms against prooxidants. Annu Rev Pharmacol Toxicol 28: 189-212, 1988. 